\title{
Límites y fragmentos en los territorios de la expansión: la suburbanización entre las ciudades de Santa Fe y Santo Tomé
}

\section{Mariana Nardelli}

Arquitecta de la Universidad Nacional del Litoral. Doctoranda en Arquitectura de la Universidad Nacional de Rosario (tesis en desarrollo: Las formas actuales de la periferia urbana. Procesos constitutivos del paisaje periférico de la ciudad de Santa Fe entre 1980 y 2010). Becaria CONICET. Docente y auxiliar de investigación en Urbanismo II de la Facultad de Arquitectura, Diseño y Urbanismo de la Universidad Nacional del Litoral. nardellimarianalis@hotmail.com

\section{Estefanía Szupiany}

Arquitecta de la Universidad Nacional del Litoral. Doctoranda en Arquitectura de la Universidad Nacional de Rosario (tesis en desarrollo: La traza y la mancha. Los corredores viales en los procesos de expansión y complejización de la estructura urbana de la ciudad de Santa Fe). Becaria CONICET. Docente en Teorías y Producción Estética en la Ciudad Moderna y Teorías y Producción Arquitectónica en el siglo XX de la Facultad de Arquitectura, Diseño y Urbanismo de la Universidad Nacional del Litoral. tefi.szupiany@gmail.com

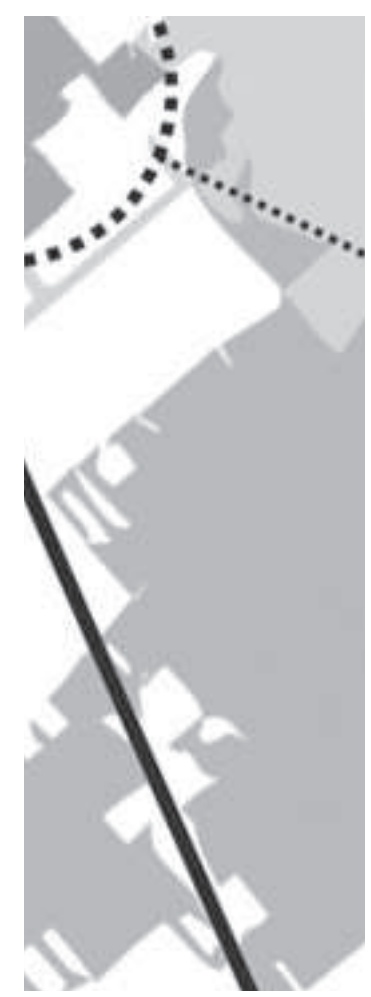




\title{
Resumen
}

Las tendencias actuales de estructuración territorial se caracterizan por procesos de fragmentación a partir de la dispersión de infraestructuras y funciones urbanas, lo que evidencia transformaciones en la organización del territorio y en los patrones de segregación de la sociedad. Las migraciones internas hacia zonas residenciales suburbanas, facilitadas por la extensión de vías circulatorias, se manifiestan entre los factores distintivos que condicionan estos cambios. En este sentido, las formas de expansión y crecimiento contemporáneo generan fragmentos socio-espaciales que pueden ser examinados a partir de la emergencia de fronteras materiales e inmateriales. Con el objetivo de analizar estos límites espaciales, temporales y sociales que materializan la fragmentación urbana, el presente estudio se focaliza en la difusión de urbanizaciones cerradas en un contexto de escala intermedia, particularmente en el caso de las ciudades de Santa Fe y Santo Tomé a lo largo de la autopista Rosario-Santa Fe.

\section{Palabras clave}

Expansión urbana; fragmentación territorial; límites urbanos; urbanizaciones cerradas.

\begin{abstract}
Suburbanization of the cities of santa fe and santo tome. Fragmentation and boundaries in the xpansion areas. The present tendencies of urban organization are characterized by processes of fragmentation due to the dispersal of infrastructure and urban functions, resulting in transformations in territorial organization and in patterns of segregation of society. Internal migrations towards residential suburban zones, facilitated by the extension of transportation networks are viewed as the distinctive factors that contribute to these changes. In this sense, the pathways of contemporary expansion and growth generate socio-spatial fragments which can be examined under the light of material and immaterial borders. The present paper focusses on the diffusion of enclosed urbanizations in a context of intermediate scale developments, particularly in the case of the cities of Santa Fe and Santo Tome, along the Rosario-Santa Fe highway, with the objective of analysing their spatial, temporal and social limits.
\end{abstract}

\section{Keywords}

Urban expansion; territorial fragmentation; urban boundaries; closed urbanizations. 


\section{Introducción}

El fenómeno de la fragmentación física y social del espacio urbano alcanzó gran parte de los territorios metropolitanos contemporáneos y orientó diversos resultados en relación con los procesos locales de urbanización. En las últimas décadas, este fenómeno dejó de ser característico de las grandes aglomeraciones y se extendió a otras escalas urbanas, de manera tal que modificó sustancialmente la configuración de ciudades de tamaño intermedio. Entre ellas se inscribe nuestro caso de estudio: la ciudad de Santa Fe y su expansión hacia las localidades próximas, particularmente el desarrollo que experimenta a lo largo de la Autopista Provincial Rosario-Santa Fe (AP 01) en la jurisdicción de la ciudad de Santo Tomé (figura 1). ${ }^{1}$

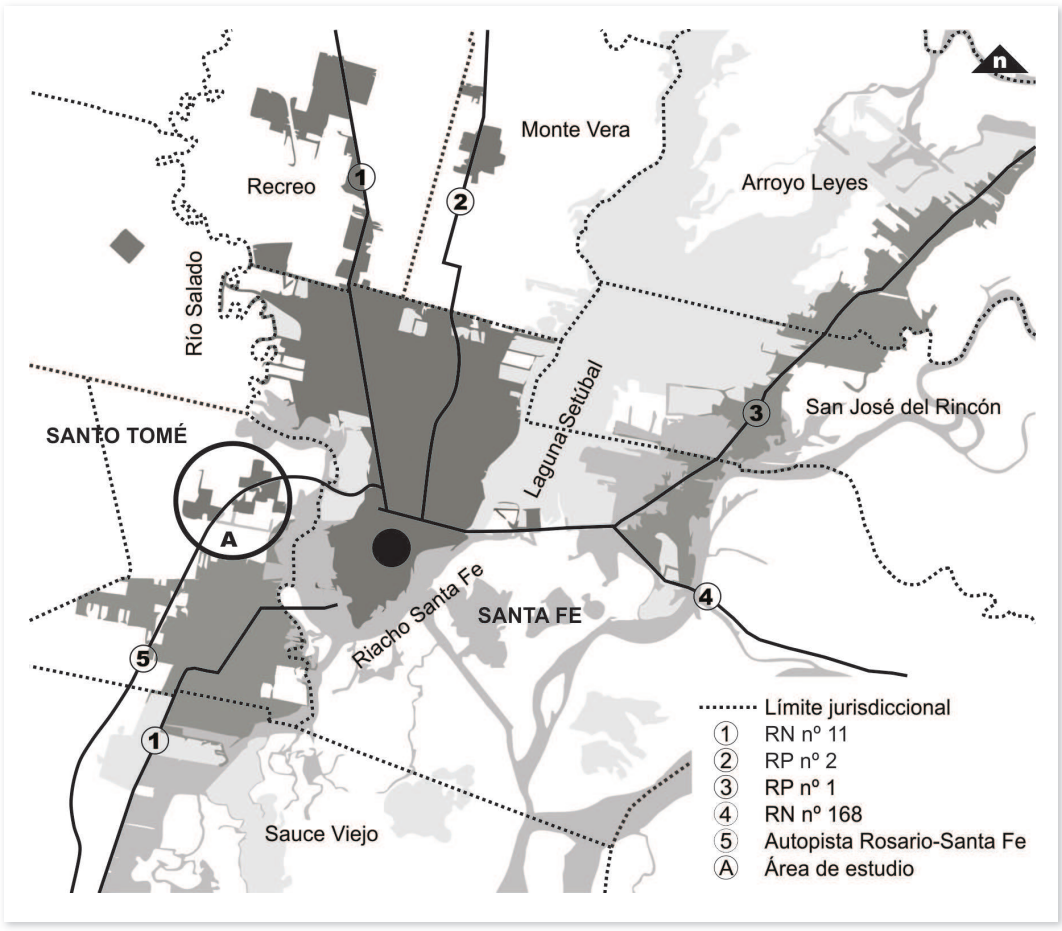

1. De acuerdo con la clasificación realizada por VAPNARSKY Y GOROJOVSKY (1990), el territorio comprendido por la ciudad de Santa Fe y sus localidades próximas, denominado Gran Santa $\mathrm{Fe}$, se inscribe en nuestro país en el marco de las Aglomeraciones de Tamaño Intermedio (ATI) Mayores, con 490.171 habitantes, según Censo Nacional de Población, Hogares y Viviendas 2010.
Figura 1. Caso de estudio en el área de expansión de la ciudad de Santa $\mathrm{Fe}$ (división por localidades) Fuente: elaboración propia a partir de datos extraídos del Servicio de Catastro e Información Territorial (SCIT) de la provincia de Santa Fe 
Más allá de las distintas conformaciones que adquieren las ciudades intermedias, existen dos tendencias dominantes y opuestas en sus procesos contemporáneos de urbanización, dos movimientos urbanos que oscilan entre lo limitado y lo ilimitado. Algunos autores hacen referencia a este par dialéctico: entornos urbanos ilimitados en sus contornos y prácticas limitadas en alguno de sus pliegues internos (CRUZ, 2013); dispersión espacial y concentración de las relaciones (SASSEN, 1991); ciudad difusa y reducción de la complejidad de la ciudad (RuEDA, 1997). Si el proceso de fragmentación consiste en la proliferación y consolidación de fragmentos urbanos homogéneos hacia su interior y heterogéneos entre sí, y en la reducción progresiva de los intercambios sociales, la noción de límite resurge en el ámbito de lo urbano, esta vez ya no en el sentido tradicional del término, es decir, contención o protección. Estos nuevos límites segregan y expulsan dentro del mismo territorio lo considerado diferente, y la ciudad ya no es lo que históricamente fue: un espacio limitado que permitía prácticas ilimitadas (CRUZ, 2013); pierde progresivamente lo que en esencia es: contacto, regulación, intercambio y comunicación (RUEDA, 1997).

El presente trabajo propone examinar la evolución y conformación actual de aquellos límites urbanos que profundizan la fragmentación territorial en el caso particular de las ciudades de Santa Fe y Santo Tomé, focalizando el análisis en las urbanizaciones cerradas dispersas a lo largo de la AP 01. A partir de esta indagación, el objetivo que aquí se postula consiste en comprender las lógicas que dominan este particular modo de ocupación del espacio urbano, al tiempo que se propone distinguir los rasgos significativos desde los cuales orientar nuevos modos de pensar el territorio. Para ello, se intentan visibilizar aquellos límites que se interponen en el desarrollo de sociedades inclusivas y heterogéneas, donde el valor de la vida urbana y la experiencia del espacio público quedan sujetos a restricciones de distintos órdenes. Las ciudades y sus expansiones están llenas de límites, y en el caso de las urbanizaciones cerradas estos no solo se materializan a través de muros y perímetros bien definidos. En los barrios privados extendidos a lo largo de la AP 01, el análisis de la discontinuidad socio-espacial permitió identificar, además de aquellos límites tangibles y fácilmente reconocibles, otros relacionados con la movilidad y el suelo urbano. Se sostiene que su reconocimiento y visibilidad son los primeros pasos inherentes al abordaje de los procesos de fragmentación del espacio urbano. 
A continuación, el escrito se organiza en tres apartados. En primer lugar, se describe y analiza el proceso general de expansión urbana de la ciudad de Santa Fe y sus localidades próximas, a partir de desarrollos fragmentarios, y se focaliza luego en el caso de estudio propuesto correspondiente a las urbanizaciones cerradas sobre la AP 01. En segundo lugar, se identifican y examinan los límites materiales e inmateriales impuestos por esta lógica segregada en la ocupación del territorio. Por último, las consideraciones finales apuntan a la importancia del reconocimiento de los límites, que tanto en el nivel urbano como en el territorial profundizan crecimientos desiguales e insostenibles, al tiempo que su identificación se considera esencial para el tratamiento de los conflictos en el marco de la seguridad, movilidad y economía urbanas.

\section{Fragmentos en la expansión urbana}

Históricamente, las ciudades han evidenciado dinámicas de diferenciación social y separación en su organización espacial (Pires Do Río CALDEIRA, 2007), que determinan patrones de segregación a partir de procesos de distanciamiento entre grupos (CAPRón, EsQuivel HerNÁNDEZ, 2015) y plasman una desigual distribución de la población en el territorio (BuRGESs, 2005). A diferencia del contexto norteamericano, donde dicho proceso sienta sus bases en atributos raciales, las ciudades latinoamericanas experimentan procesos segregatorios que espacializan las condiciones sociales a partir de criterios socioeconómicos o de clase (CARMAN, Vieira y SEgURA, 2013).

En este sentido, los patrones de segregación han ido expresando sus formas en los diferentes modos organizativos del espacio. Mientras que en la ciudad tradicional, concentrada, el distanciamiento social se concretaba por tipo de vivienda, en el modelo centro-periferia es la distancia la que divide a la población en sectores sociales (Pires do Río CALDEIRA, 2007). En las últimas décadas, las reglas de estructuración y expansión urbana han mutado la organización territorial en los niveles morfológico, social y funcional de las ciudades.

Una de las tendencias actuales en la estructuración del territorio se caracteriza por el principio de fragmentación, que determina la dispersión de infraestructuras y funciones urbanas. ${ }^{2}$ En efecto, las lógicas del modelo centro-periferia se superponen a una organización del espacio en el cual los grupos sociales se sitúan próximos pero se encuentran separados
2. Esta estructura organizativa del espacio urbano conforma, junto a otros conceptos (Ciudad Difusa, periurbanización, rururbanización, metápolis, suburbia, contraurbanización, exópolis, edgies cities, megalópolis, entre otras) una de las teorías explicativas dentro de las discusiones que pretenden dar cuenta de los fenómenos urbanos contemporáneos. 
3. Mientras las ciudades centrales como Santo Tomé y Santa Fe presentan una variación intercensal del $122.4 \%$ en el primer caso y $49.86 \%$ en el segundo, siendo las ciudades con menor crecimiento para el período 1960/1980 y 1991/2010, las localidades próximas como Recreo $(458.88 \%)$, Sauce Viejo

(441.66 \%), Arroyo Aguiar (358.44\%), San José del Rincón (250\%) y Monte Vera $(247.64 \%)$ evidencian un crecimiento relevante para el mismo período.

4. Para más información ver COLLADO (2012), BAGNERA (2010), RAUSCH (2005). por muros o barreras. Este modelo refiere a un proceso espacial de obstáculos físicos (BuRGESS, 2005), tanto de edificación como de ocupación del suelo. Así, plantea dependencia relativa a los centros urbanos, pero con discontinuidades y ausencia de contigüidad tanto en la trama como en el tejido de dichos centros (Salinas Varela, 2009). En este contexto, el modelo urbano fragmentado que adquieren algunas ciudades - particularmente en Latinoamérica- se caracteriza por un crecimiento a partir de nodos o fragmentos y la introducción de cercos y barreras (BoRSDORFT, 2003), que representan una discontinuidad con el sistema de espacios públicos propios de la ciudad tradicional.

En la ciudad de Santa Fe y sus localidades próximas, la manifestación de estos procesos en el espacio urbano evidencia formas de expansión expresadas en la dispersión de funciones a partir del desarrollo de las infraestructuras de conexión territorial. La lógica dominante en la localización de actividades responde a la rápida accesibilidad y cercanía a las vías de comunicación vehicular, al tiempo que denota procesos de crecimiento cada vez más dependientes de la automovilidad. En este sentido, la estructuración urbana en función de los ejes viales impulsa un proceso simultáneo tanto en la distribución de actividades específicas como en la expansión residencial por fuera del núcleo urbano central, y adquiere ciertas particularidades en el desarrollo general. ${ }^{3}$ Así, sobre la expansión hacia el este se localizan, en su primer tramo sobre la Ruta Nacional 168 (RN 168), actividades especializadas relacionadas con la vida académica y científica de la región, como así también actividades recreativas y de consumo. En el segundo tramo sobre la Ruta Provincial 1 (RP 1) se consolidó el llamado "corredor de la costa" a partir de la residencia con una fuerte caracterización del paisaje costero. ${ }^{4}$ Por su parte, sobre la extensión de la Ruta Nacional 11 (RN 11), tanto en dirección norte (ciudad de Recreo) como en dirección sur (ciudad de Santo Tomé y comuna de Sauce Viejo) se configuró el cordón productivo e industrial a partir de la instalación de fábricas, depósitos de almacenamiento, parque industrial, producción frutihortícola, entre otras. Hacia el oeste, en proximidad a la AP 01, el crecimiento se apoya exclusivamente a partir de la instalación de clubes de campo y barrios cerrados. Estos últimos se configuraron a modo de nodos homogéneos y determinaron procesos fragmentarios en la estructuración general del territorio.

Al tiempo que estas extensiones se consolidan en torno de la red vial principal, la dinámica general del continuo urbano mantiene una concentración de actividades en el área central 
Limites y fragmentos en los territorios de la expansión:

la suburbanización entre las ciudades de Santa Fe y Santo Tomé

de la ciudad de Santa Fe, donde se localizan gran parte de los servicios, comercios, equipamientos y empleos. Dinámicas centrípetas que profundizan la interdependencia de actividades laborales y administrativas (entre las de mayor relevancia) con la ciudad central. El crecimiento poblacional de los últimos períodos impulsó la expansión del área urbanizada y generó cambios en la estructura territorial.

Las urbanizaciones cerradas como

impulsoras de la fragmentación urbana

En el marco de las reconfiguraciones territoriales a partir de modelos de estructuración por fragmentos, los enclaves cerrados se consolidan entre una de las lógicas espaciales que agudizan los procesos de segregación socioespacial. Algunos autores interpretan estas formas urbanas en tanto motores de transformación de la ciudad contemporánea (JANOschKA, 2002), y las definen a partir de límites materiales e inmateriales que adquieren carácter físico, social, temporal y económico (CAPRon y EsQuivel HernÁNDEZ, 2016). Entre las manifestaciones de esta configuración, las urbanizaciones cerradas suburbanas emergen de la trama urbana a partir de su localización aislada y discontinua, aunque siempre próxima a alguna autopista o vía rápida de comunicación con el centro de la ciudad. Sus rasgos característicos - seguridad y vigilancia privada, modificación de hábitos sociales, menor presencia de esferas públicas - pueden ser interpretados en el marco de una práctica de auto-segregación, según la cual los habitantes se perciben a sí mismos como vulnerables (CARMan, VieIRA y SEGURA, 2013). Por su parte, Borsdorf (2003) sostiene que estas urbanizaciones permiten comprender las lógicas actuales de configuración territorial, y con ello interpretar las pautas culturales que desarrollan las sociedades. Así, los discursos del miedo y la seguridad, el deseo de una vida vinculada con la naturaleza y alejada del caos urbano generan un modelo de segregación basado en valores de desigualdad, separación y control. Estos enclaves alteran la concepción tanto del espacio como del desarrollo de la vida en el espacio público, anulan la diversidad de los intercambios y promueven la búsqueda de un entorno social homogéneo (JANOSCHKA, 2002).

Esta escisión materializada en el espacio urbano aflora en un contexto de pérdida del control estatal en la regulación del uso del suelo, condicionado por la implementación de políticas neoliberales en América Latina y, particularmente en Argentina, desde los años 90. Al 
5. Las urbanizaciones cerradas de Nordelta representan un caso paradigmático en Argentina. En una superficie total de 1600 hectáreas se concentran veinte barrios cerrados construidos por el mismo promotor inmobiliario

(JANOSCHKA, 2004).

6. El complejo La Tatenguita (1976) y el Club de Campo el Paso (1982) fueron las primeras urbanizaciones de este tipo. Años más tarde, se sumaron El Pinar (1997), Dos Lagunas (1999), Las Almenas (2009), Aires del Llano (2009) y Altos de la Ribera (2013).

7. Luego de la derogación de las Ordenanzas $N .^{\circ}$ 2390 en 2003 y $\mathrm{N}^{\circ} 2843$, actualmente se encuentra vigente la Ordenanza $N .^{\circ}$ 3069, sancionada en 2014 que incorpora la figura del condominio dentro de las urbanizaciones cerradas. En 2011, a partir de la Ordenanza $N .^{\circ} 2844$ de regulación de usos del suelo, se delimitó un espacio para las DUE (Distrito de Urbanizaciones Especiales) dentro de la planta urbana (PALLUD, 2010). tiempo que se agudizaron las desigualdades socioeconómicas, nuevas lógicas de mercantilización del suelo modificaron el patrón tradicional de segregación socioespacial a partir de un masivo efecto diferenciador sobre la estructuración del territorio urbano (REESE, 2007). En este sentido, CuENYA (2016) alude a la noción de laissez faire para explicar la desregulación del mercado del suelo en la producción de countries y barrios privados, orientada por políticas urbanas que priorizaron los intereses del capital. Estas afirmaciones evidencian la sustitución del Estado por parte de fuerzas económicas focalizadas en una mayor rentabilidad de sus inversiones inmobiliarias. ${ }^{5}$ En este sentido, Svampa (2004) sostiene que la fractura social se tradujo en formas específicas de segregación, entre la emergencia de nuevos ghettos de pobreza y entre aquellos actores sociales atentos a las nuevas reglas del juego económico. De estos últimos, y en relación con el surgimiento de las urbanizaciones cerradas como producto inmobiliario, la autora destaca la figura del desarrollador urbano en tanto actor clave para comprender la difusión de un discurso que legitima los beneficios de un nuevo estilo de vida acorde con la demanda de las clases dominantes.

Esta lógica mercantilista que dio origen a la formación de urbanizaciones cerradas en áreas suburbanas se evidencia en la expansión hacia el oeste de la ciudad de Santa Fe, sobre la jurisdicción de la ciudad de Santo Tomé y en torno del eje de la AP 01 (figura 2). Si bien las primeras construcciones datan de la década del 70, no es sino hasta los años 90 cuando se manifiesta una ocupación acelerada que mantendría su impulso hasta la actualidad. ${ }^{6}$

En sus inicios, estas urbanizaciones se concretaron de manera irregular, fuera de un marco jurídico adecuado, ya que el municipio adoptó normas propias de otros contextos y situaciones urbanas. ${ }^{7}$ Fueron los desarrolladores inmobiliarios privados quienes impulsaron estas urbanizaciones, llevaron con ello infraestructura y servicios al sector y apelaron a discursos de promoción enfocados en la naturaleza como valor agregado. Años más tarde, resuelto el marco normativo para regir este tipo de ocupaciones, la promoción de los nuevos barrios incorporó en su discurso la posibilidad de escrituración inmediata, sumada a los discursos previos relacionados con la seguridad y la vida natural.

A continuación, se propone una lectura analítica de los límites tangibles e intangibles que materializan la fragmentación espacial en el caso de estudio propuesto, a fin de comprender las dinámicas contemporáneas que condicionan las formas de expansión urbana y territorial. 


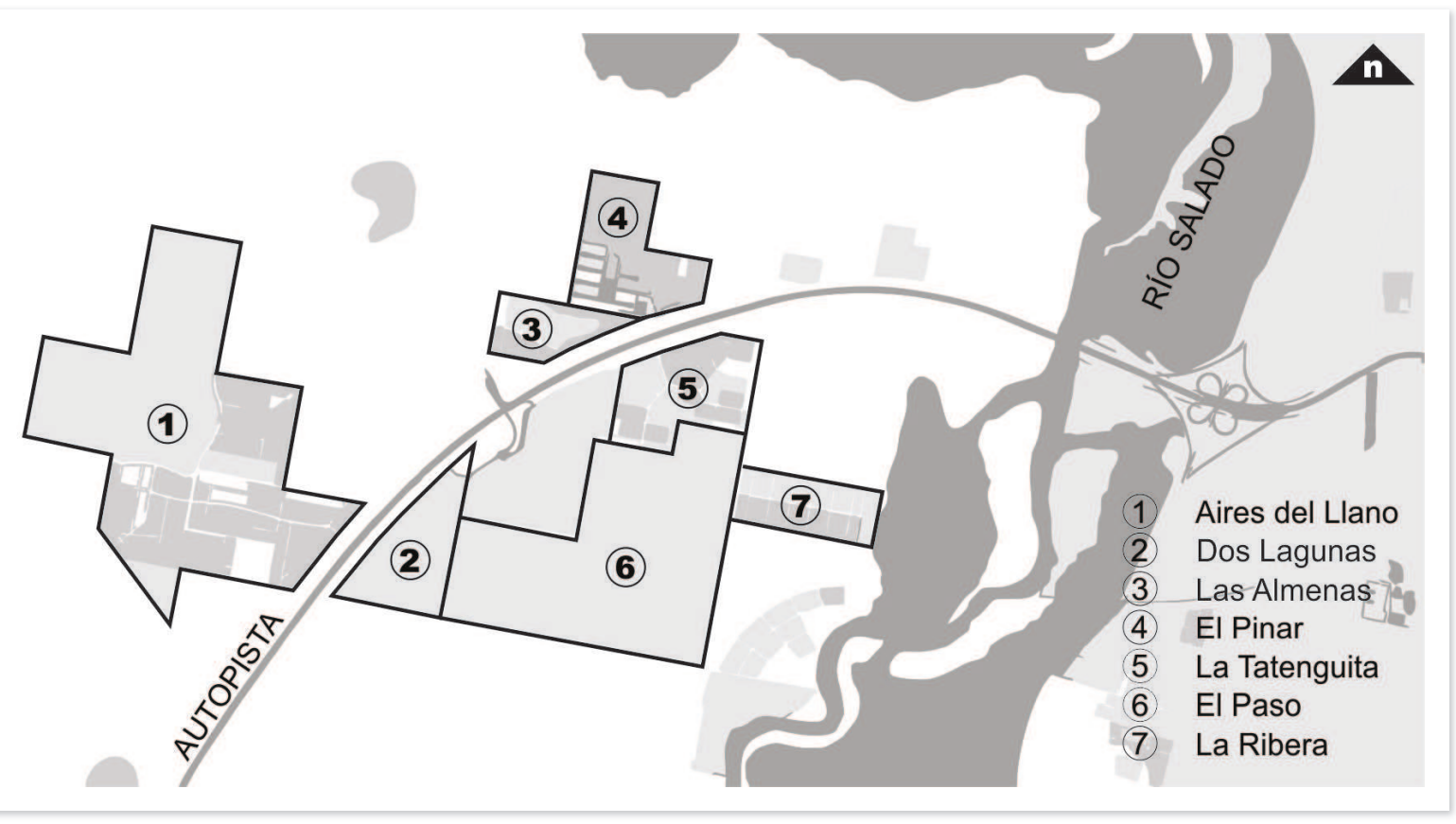

Límites que fragmentan: tres modos de evidenciar las fracturas en la estructura interna de la ciudad

La dispersión de fragmentos en áreas de expansión del tejido urbano supone prácticas generalmente monofuncionales (educación, comercio, industria, residencia, etc.) que tienden a diluir la complejidad en la mayor parte del territorio (RUEDA, 1997). Cada uno de esos enclaves imponen limitaciones, no siempre conscientes y planificadas, al libre acceso e intercambio de actividades y personas, y por lo tanto el único espacio abierto y público más cercano lo constituyen los caminos, rutas o autopistas desde donde se distribuyen estos fragmentos aislados entre sí. En los enclaves residenciales cerrados esta imposición de límites, a dife-
Figura 2. Urbanizaciones privadas en la jurisdicción de la ciudad de Santo Tomé. Fuente: elaboración propia a partir de datos extraídos del Servicio de Catastro

e Información Territorial (SCIT) de la provincia de Santa Fe 
rencia de otros fragmentos, son determinaciones conscientes sometidas a la elección por la autoexclusión. Los distintos impedimentos al intercambio social y al contacto con lo público que allí se construyen tienen que ver con barreras tangibles e intangibles que actúan como filtros en los procesos de admisión. En este sentido, MARcuse (1995: 84) argumenta: "Quiero utilizar muros -límites, particiones, fronteras, transiciones - a la vez como una corporeidad y una metáfora para la naturaleza de estas divisiones sociales, muros como reflexión y como refuerzo, al mismo tiempo, de las divisiones".

En el estudio de las urbanizaciones cerradas localizadas al suroeste de la ciudad de Santa Fe y pertenecientes al municipio de Santo Tomé, se identificaron los límites materiales e inmateriales que desvinculan el espacio urbano y disminuyen los intercambios entre distintos grupos sociales. Este reconocimiento se concretó a partir del análisis y observación de un conjunto de datos empíricos (infraestructuras, accidentes topográficos, cercos materiales, accesibilidad), a través de recorridos fotográficos e imágenes satelitales. Al mismo tiempo, se sistematizaron datos de tipo censal, tránsito medio, parque automotor, unidades de vivienda y se indagó en crónicas periodísticas y publicidades inmobiliarias. A partir de este relevamiento se determinaron tres tipos de límites en relación con el modo en que dichas barreras son impuestas. Nos referimos a los límites espaciales (materiales e inmateriales), temporales (distancia y automovilidad privada) y sociales (mercantilización y acceso al suelo urbano).

\section{Límite espacial}

La discontinuidad espacial del entorno urbano puede ser afectada por barreras físicas naturales o artificiales. En el caso de las ciudades de Santa Fe y Santo Tomé, el curso del río Salado y su valle de inundación las separan en un ancho variable entre los 1300 y $2000 \mathrm{~m}$, aproximadamente. La conexión física está resuelta a través de dos cruces viales: el puente Carretero, inaugurado en el año 1939, y el puente de la AP 01, localizado cinco kilómetros al norte del anterior y construido a inicios de la década del 70. Dado este fuerte condicionante al crecimiento de dichas ciudades impuesto por el cauce del río, la expansión del tejido urbano se ha ido consolidando de manera diferencial a lo largo de las vías circulatorias continuas a ambos puentes. Precisando el caso de la ciudad de Santo Tomé, el desarrollo de su planta urbana se originó, desde su fundación en 1872, en la zona norte entre el puerto y 
el ferrocarril, y se extendió hacia el sur en la década de 1940 a partir de la pavimentación de las rutas nacionales 11 y 19 (PALLUD, 2010).

A mediados de la década del 90, el fenómeno de las urbanizaciones cerradas comienza a desarrollarse a la vera de la AP 01 y genera un nuevo polo de crecimiento hacia el norte de la ciudad. Así, mientras el desarrollo de la ciudad de Santo Tomé con sus barrios tradicionales se consolidó en función del puente Carretero, las urbanizaciones ubicadas sobre la autopista corresponden al surgimiento de nuevas formas de ocupación del territorio.

Analizando sus patrones de configuración, resulta posible evidenciar que estas urbanizaciones establecen una doble limitación a partir de su localización. Por un lado, el cauce del río Salado, en tanto barrera natural, posibilita la desvinculación de estos enclaves respecto de la población con mayor vulnerabilidad social de la ciudad, situada sobre el margen derecho. Por el otro, el alejamiento físico con el núcleo urbano central establece una distancia tanto espacial como social en relación con los habitantes de la ciudad. Sin embargo, se plantean diferencias respecto de casos de ciudades de mayor escala, como San Pablo (PIRES Do Río CALDEIRA, 2007), Buenos Aires (JANoschKA, 2002) o Santiago de Chile (Hidalgo, 2004). En ellas, se superpone dicho patrón con un modelo que introduce barreras - muros y dispositivos de seguridad y vigilancia privada (Pires do Río CALDEIRA, 2007)— que lo separan del entorno inmediato, generalmente de áreas sociales degradadas. La particularidad del caso de estudio supone que estos asentamientos se implantan aisladamente en un entorno suburbano sobre el cual no limitan más que con las mismas urbanizaciones. Así, se establecen dinámicas de fragmentación espacial en tanto desarticulación del espacio urbano, aunque con una segregación socioespacial atenuada, en tanto que los grupos sociales que componen sus entornos inmediatos corresponden a otros barrios cerrados.

Más allá del límite natural entre ambas ciudades, existen límites físicos artificiales que interrumpen las actividades urbanas tradicionales y, por lo tanto, el intercambio y la continuidad socio-espacial. Al igual que gran parte de las urbanizaciones privadas en otros contextos, ${ }^{8}$ los barrios cerrados emplazados al norte de la ciudad de Santo Tomé presentan perímetros bien definidos, generalmente materializados por tejidos metálicos, alambres de púa, cercos verdes o muros de mampostería. Cada uno de estos limita y separa el espacio público del privado. Separa modos de vida y clases sociales. En algunos casos, los límites perimetrales presentan casi rítmicamente torres de control, con el objetivo de impedir cualquier intrusión física extraña
8. De igual modo que en otros países latinoamericanos como México (CAPRÓN GUENOLA, ESQUIVEL HERNÁNDEZ, 2015), Brasil (PIRES DO RIO CALDEIRA, 2007) o Chile (HIDALGO, 2004), las urbanizaciones cerradas resultan coincidentes en tanto concentran población con niveles socioeconómicos medios y altos, por fuera de la aglomeración urbana, y utilizan tecnologías de exclusión social como medio de separación de barrios tradicionales urbanos. 
9. Este rechazo al externo sospechoso fue experimentado durante el trabajo de campo. El personal de seguridad vigilo nuestro recorrido sobre la vía pública que rodea las urbanizaciones cerradas, evidenciando su deber de protección y control. Una experiencia similar queda registrada en el documental

"La ciudad que huye", de Lucrecia Martel.

10. En el interior de estos barrios no existe división entre lotes a partir de cercos materiales, condición que junto a ciertas reglamentaciones de construcción en la definición tipológica de las viviendas otorga homogeneidad y continuidad en sus configuraciones estéticas y arquitectónicas. o ajena al barrio (figura 3). MARcuse (1995) denomina a estos límites "muros de estuco", protegen a las comunidades exclusivas y excluyen por razones de estatus y control social. ${ }^{9}$ Esta definición de borde es siempre acompañada por pórticos de acceso restringidos y seguridad privada, donde se realizan las tareas de selección y control de los admitidos.

El carácter monofuncional del sector urbano donde se agrupa este tipo de urbanizaciones torna necesario el vínculo funcional con las ciudades próximas, vinculación materializada en su localización estratégica respecto de los ejes de circulación. Las actividades laborales, educativas y de salud se concentran en las ciudades de Santa Fe y Santo Tomé, y por lo tanto la interdependencia a pesar de la distancia prevalece. Esta homogeneidad funcional, junto a la delimitación física de cada uno de los barrios, implica la renuncia a todo tipo de intercambio social y dinamismo cotidiano, y privilegia el aislamiento y la individualidad por sobre los valores colectivos. Este retraimiento se hace visible en las desoladas calles circundantes, flanqueadas por barreras que utilizan el recurso de lo verde y lo natural para ocultar su perversidad y agresión a lo público. Paradójicamente, hacia el interior se observan artificios de comunidad y continuidad espacial al desaparecer los límites que tradicionalmente delimitan los lotes y las viviendas particulares, ${ }^{10}$ una concepción que, según JANoschKA (2004), se comprende como la contraparte del caótico y no planificado mundo exterior. Al mismo tiempo que el muro representa cierta pertenencia, rodea resguardando del peligro y excluyendo cualquier extraño a estas urbanizaciones. De este modo, se entiende que la fragmentación no es aleatoria: residir en enclaves cerrados se vuelve una elección (MARcuse, 1995). En este sentido, CABrales Baraja y Canosa Zamorra (2001) entienden que las urbanizaciones cerradas instan a replantear la idea tradicional de ciudad al ser planteada una anticiudad. Los límites encierran la vida paradisíaca que deja por fuera de ellos el caos de la ciudad actual, y la seguridad se torna un bien adquirible que no puede ser combatido desde la esfera pública.

\section{Límite temporal}

La expansión materializada en las urbanizaciones cerradas a lo largo de la AP 01 se aparta y aísla respecto de la trama urbana de las ciudades de Santa Fe y Santo Tomé. Así, el incremento de las distancias, la necesaria movilidad diaria hacia el centro de ambas ciudades y la inaccesibilidad del transporte público a estos barrios condicionan la segunda limitación que aquí se aborda: la posibilidad de acceso a la automovilidad privada. 

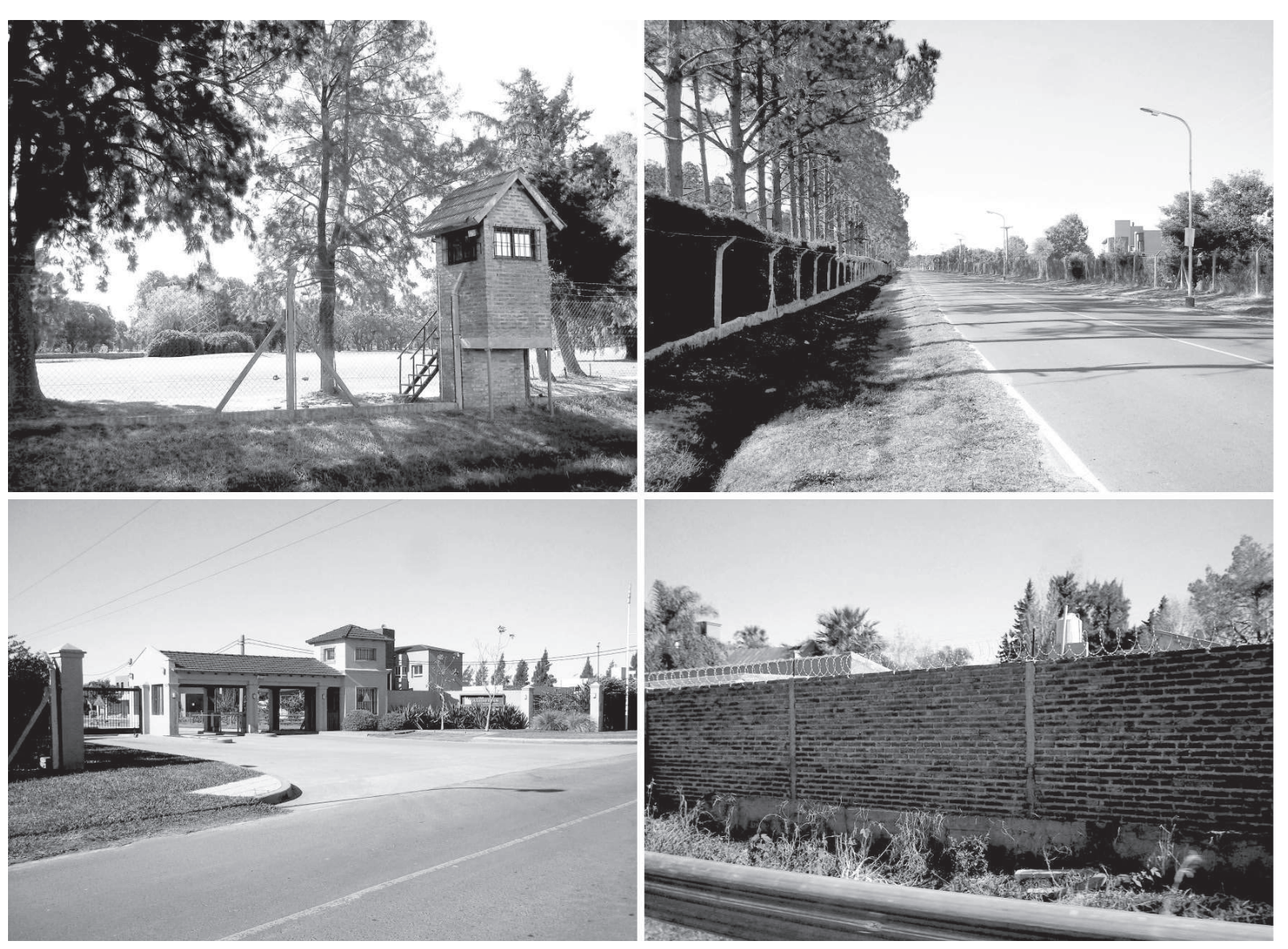

Figura 3. Límites físicos en las urbanizaciones privadas de la ciudad de Santo Tomé. Fuente: fotografías de autoras 
11. "Esta obra [...] marca una etapa fundamental en

la historia de la Vialidad Argentina: representa la incorporación de un medio moderno, de gran aliento, en los sistemas viales provinciales. [...] Podría afirmarse que estas obras habrían merecido la más profunda afirmación y el más enfático apoyo de Sarmiento, el gran civilizador de la Argentina, porque ellas son eficaces instrumentos del desarrollo y progreso general” (ASOCIACIÓN ARGENTINA DE CARRETERAS, 1966: 29-30).

12. Datos extraídos de la Dirección Provincial de

Vialidad de la Provincia de Santa Fe en relación con el Tránsito Medio Diario Anual (TMDA) revelan que en el período 1995-2015 el número de automóviles que circulan sobre la AP 01 en el tramo Santa Fe-Santo Tomé es de 6877, mientras que ese número se reduce a 3120 en el tramo Santo Tomé-Sauce Viejo.
La construcción de autopistas a partir de la década del 60 en Argentina favoreció la radicación del fenómeno country en ciudades donde aún no se registraban clubes de campo. Por aquellos años, la provincia de Santa Fe, por intermedio de la Dirección Provincial de Vialidad, emprendió la autopista Rosario-Santa Fe. Su importancia queda revelada en crónicas de la época. ${ }^{11}$ El optimismo en torno a la nueva autopista no tardó en ser aprovechado por un sector social deseoso en imitar la experiencia de los clubes de campo existentes en otras ciudades argentinas. El Complejo La Tatenguita en el año 1976 y el Club El Paso en 1982 iniciaron un proceso de transformación en las lógicas de ocupación del territorio santafesino. El trazado de la autopista representaba "... la posibilidad de adquirir su fin de semana $o$ su vivienda - ¿por qué no si está distante solo 3500 metros de la ciudad?” (DiARIo El Litoral, 1975). Esta crónica enfatiza la ubicación de la nueva urbanización no solo a partir de su rápida accesibilidad, además, pone en discusión el nuevo emprendimiento respecto de las formas existentes de suburbanización: "La ubicación de este conjunto residencial deportivo es ideal. Es lindante con la autopista y está ubicado 4.5 kilómetros al norte del área urbana de Santo Tomé, a 3.5 kilómetros de nuestra ciudad, es decir que para los santafesinos es mucho más cercano que Guadalupe, Rincón, Arroyo Aguiar y cualquier otro lugar cercano que pudiera considerarse excelente para los fines expuestos. Los accesos al lugar son fáciles por los caminos rurales de la zona y tiene acceso directo a la autopista sin que se desvirtúen las características de esta útil vía de comunicación” (Diario El Litoral, 1975).

Concebida así como eje de distanciamiento, la autopista se convierte en una limitación al intercambio social, privativa para quienes pueden acceder a la automovilidad privada. En referencia a este cambio en la forma de percibir el espacio urbano, JANoschKA (2004) argumenta que el masivo proceso de suburbanización y los cambios en las modalidades de viaje modificaron sustancialmente tanto la percepción de la distancia como la percepción comunitaria de la sociedad. Esta transformación se vio favorecida por el acortamiento del tiempo de movimientos pendulares diarios que ofrecían las ubicaciones estratégicas respecto de la autopista, lo que modificó la percepción de la distancia. ${ }^{12}$ Como puede verse en la figura 4 , la promoción de estas áreas residenciales ya no radica solo en los discursos relacionados con la naturaleza, exclusividad y seguridad, aquí el tiempo se incorpora como factor de atracción para la venta. En este sentido, CACCIARI (2005: 56) sostiene: "Se trata de territorios y habitamos territorios cuya métrica ya no tiene ningún sentido espacial, sino, solo en el mejor de los casos, temporal. Hacemos todas nuestras cuentas en base al tiempo, no al espacio; ya nadie indica la distancia a la que se encuentra una ciudad, sino el tiempo que se tarda en llegar a ella. El espacio se ha convertido únicamente en un obstáculo”. 
Mientras las autopistas constituyen el soporte para el acortamiento del tiempo de los desplazamientos cotidianos y, por lo tanto, de una nueva percepción de la distancia, el automóvil particular constituye el medio a partir del cual se modifica la percepción comunitaria de la sociedad. Para quienes habitan los barrios cerrados distantes al centro de la ciudad, la movilidad vehicular propia se convierte en el único medio de traslado posible, en detrimento del transporte público y de los espacios urbanos accesibles a toda la comunidad. El intercambio y los contactos se reducen al mínimo, y esto deriva en una nueva manera de percibir el espacio y la sociedad (JanoschKa, 2004), o, como afirma RuEda (1997: 10), en "nuevos retrocesos de la capacidad autónoma para desplazarse". En este contexto, la distancia comienza a ser entendida como posibilidad de acceso y, al mismo tiempo, lejanía de aquello que se pretende no tener cerca. De este modo, la fragmentación se ve representada en el tiempo que anula las distancias para los que así lo eligen, aísla del diferente, protege el privilegio. En este sentido, la separación física materializada a partir de la distancia genera segregación social al pretender reunir en determinadas áreas atributos similares, poblaciones homogéneas, convertir las barreras físicas en barreras sociales y, con ello, profundizar las restricciones al intercambio e integración socio-espacial.

\section{Límite social}

Entre los factores más importantes en la consolidación de urbanizaciones cerradas en áreas de expansión urbana, es determinante la capacidad de acceso al suelo en función de los niveles de renta que poseen los distintos grupos sociales. La debilidad del Estado para intervenir en la planificación del territorio y la fuerte incidencia del mercado inmobiliario profundizan lo que Svampa (2004) denominó “urbanismo de afinidades”, según el cual la búsqueda creciente de homogeneidad social está acompañada por la oferta diferencial de suelo urbano.

Por su parte, REESE (2007) caracteriza esta nueva forma de segregación voluntaria en tanto producto inmobiliario que promueve la suburbanización de determinados grupos sociales, e inserta la problemática en el marco del amplio debate sostenido en torno a las políticas de suelo en América Latina. Los cambios registrados en los patrones de producción y configuración de las ciudades, sostiene REESE, han convertido el manejo de la tierra en un factor crítico para enfrentar los desafíos y conflictos emergentes de las nuevas dinámicas urbanas, entre ellos, los efectos 


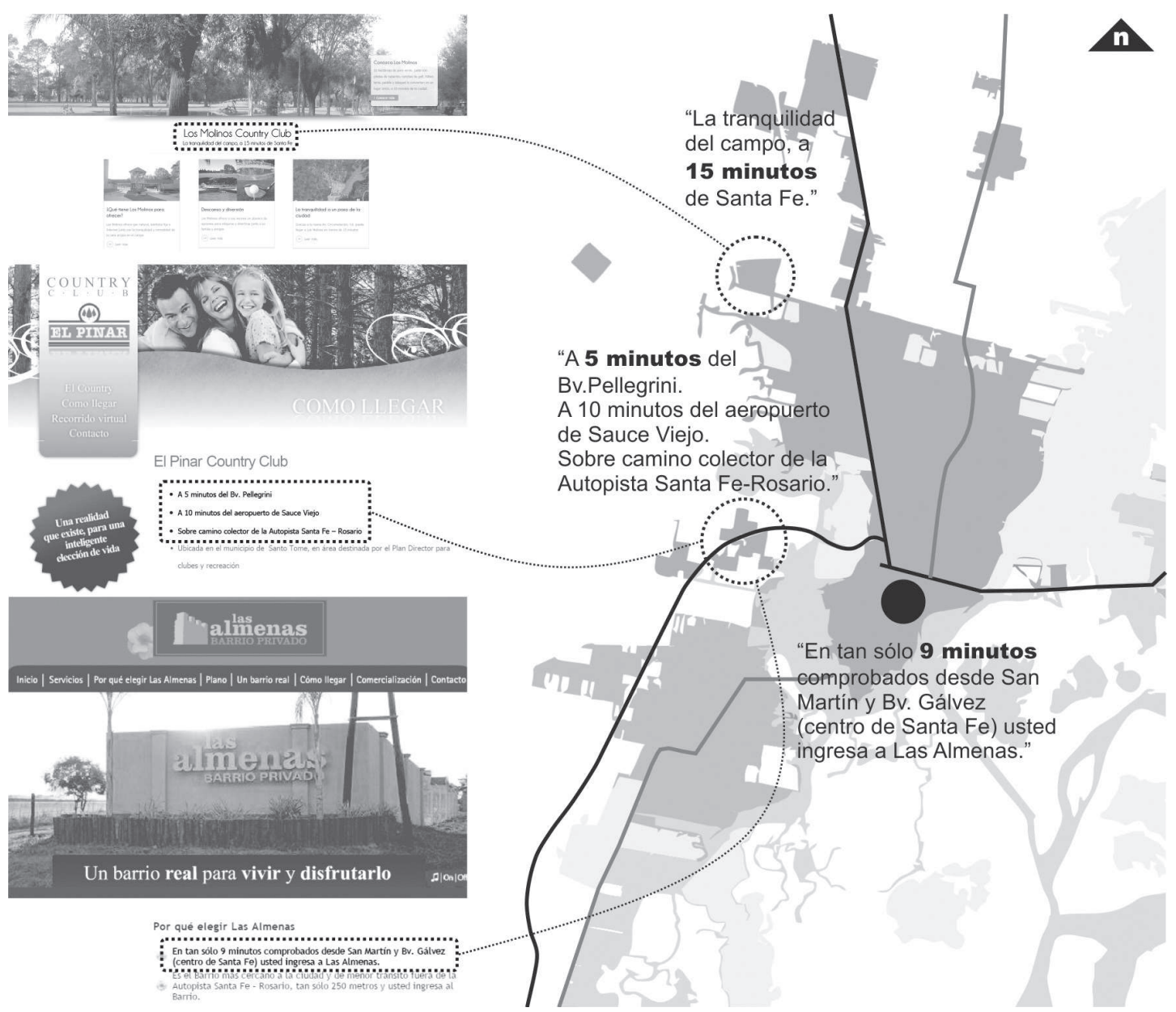

Figura 4. Discursos de promoción con énfasis en el tiempo de recorrido al centro urbano.

Fuente: elaboración propia a partir de publicidades digitales de urbanizaciones cerradas (Los Molinos country club, country club El Pinar y Las Almenas barrio privado) 
negativos producidos por el funcionamiento del mercado del suelo. Así, esta oferta diferencial asume procesos de mercantilización —mayor protagonismo del capital inmobiliario-y elitización — conformación de nuevas élites de consumidores— de la ciudad (CUENYA, 2016).

NÉSTOR GómEZ (2011), en su trabajo dirigido a la segregación socio-residencial en la ciudad de Santa Fe, entiende que las posibilidades de elección del ámbito de residencia por parte de los distintos sectores sociales se determinan a partir de sus capacidades en relación con el nivel de instrucción y empleo. Estos factores permiten a los grupos con mayores ingresos elegir su lugar de residencia - generalmente en zonas servidas, con mayor calidad de infraestructura y equipamientos, y de seguridad frente al riesgo hídrico-, condicionando a quienes no poseen las mismas posibilidades de elección a localizarse en áreas con carencias urbanas y vulnerabilidad hídrica y social.

En el caso del territorio santafesino, el fenómeno de la fragmentación deviene de un proceso histórico de construcción desigual, condicionado por una distribución espacial de los grupos sociales. En términos generales, el centro quedó a disposición de los sectores con condiciones económicas más favorables, y la población de menores recursos fue desplazada hacia el oeste y noroeste de la ciudad, donde el valor inmobiliario presenta los menores índices de tasación. En los últimos años, la consolidación de los emprendimientos residenciales de características cerradas en proximidad a la AP 01 complejizó y profundizó los contrastes de esta fragmentación. La debilidad regulatoria por parte del Estado para intervenir en la conformación de estos fragmentos segregados, junto con la incapacidad para ofrecer condiciones de vida favorables a los sectores más vulnerables, se traduce en el advenimiento de nuevos límites y fronteras.

En el caso local, este proceso de homogeneización social a partir de la formación de barrios cerrados cobra cierta particularidad en el marco del Programa de Crédito Argentino del Bicentenario para la Vivienda Única Familiar (Procrear) lanzado en el año 2011 por el gobierno nacional. El origen de las urbanizaciones cerradas en Santo Tomé data del período 1970-1980, pero su proceso de ocupación se aceleró en la década del 90, cuando las políticas neoliberales implementadas por el nuevo modelo económico del gobierno nacional ofrecieron al mercado inmobiliario mayores libertades y concesiones, y permitieron a los desarrolladores la creación de un nuevo producto urbano destinado a la población con mayores ingresos. ${ }^{13}$
13. Datos obtenidos de Catastro Provincial revelan estos incrementos sucesivos de las unidades de vivienda: entre 1978 y $2001 \mathrm{el}$ aumento fue de $10,63 \%$ (105 uv), y se observa un crecimiento gradual de $15,69 \%(155 \mathrm{uv})$ entre 2002 y 2005, y de $20,45 \%$ (202 uv) entre 2006 y 2010 Un aumento relevante se evidencia entre 2011 y 2014 al incrementarse en un $50,24 \%(526 u v)$ 
Mariana Nardelli y Estefania Szupiany

14. Desde el lanzamiento de los créditos en el año 2011, se evidencia que en la ciudad de Santa Fe el impacto de los créditos representa el $31,10 \%$ en el período 2013-2015, mientras que en Arroyo

Leyes la incidencia de los créditos fue del 77,72\% para el mismo período.

15. Datos provenientes de Catastro Provincial, Santo

Tomé.
A partir del lanzamiento del crédito argentino Procrear, esta oferta se extendió a la clase media. La búsqueda de terrenos aptos a ser escriturados y con las condiciones urbanísticas requeridas para la hipoteca acentuó la expansión del tejido residencial hacia las localidades vecinas de Santa Fe, principalmente en Recreo, Monte Vera y Arroyo Leyes. ${ }^{14}$ En el caso particular de la ciudad de Santo Tomé, el impacto de estos créditos impulsó la construcción de nuevas viviendas en los barrios cerrados existentes que aún mantenían lentos procesos de urbanización. Datos registrados en noviembre de 2012 a septiembre de 2013 evidencian que de un total de 134 permisos de edificación, 68 se construyeron en la zona urbana de la ciudad de Santo Tomé y 66 en las urbanizaciones cerradas, es decir que mientras el 50,75 \% se construyó en la ciudad, un 49,25\% se concretó en estos espacios segregados. ${ }^{15}$ A partir de estos datos se comprende que la fuerza impulsora por la que determinados grupos sociales elijen radicarse en estos barrios ya no responde solo la búsqueda de exclusividad, seguridad y naturaleza. A estos argumentos se suma la posibilidad de adquirir lotes adaptables a los requisitos impuestos por el crédito. Ante la incapacidad del Estado para generar suelo urbano y con la lógica de laissez faire (CUENYA, 2016), el mercado inmobiliario asume esta responsabilidad y la convierte en oportunidades económicas, autorizadas y facilitadas por las entidades municipales.

\section{Consideraciones finales}

La importancia de examinar los límites urbanos que dividen y fracturan la estructura interna de las ciudades, atentando contra la continuidad e intercambio socioespacial de los entornos urbanos, radica en la necesidad de abordar el problema de la fragmentación urbana a partir de los elementos materiales e inmateriales que originan y profundizan el fenómeno. Se trata, por lo tanto, de un aporte metodológico a partir de la identificación de las dinámicas en juego. Las limitaciones que aquí se exponen son manifestaciones de la organización política y social del territorio y, por lo tanto, su análisis permite comprender los factores que dan lugar a procesos desiguales de urbanización. En consecuencia, a partir de la caracterización expuesta de los límites espaciales, temporales y sociales en el marco de esta emergencia y difusión de fragmentos sobre el territorio, se desprenden algunas líneas de discusión que permiten comprender los procesos en los que nuestras ciudades se encuentran inmersas. Al mismo tiempo, estas reflexiones pueden convertirse en supuestos claves para la ordenación y planificación urbana en el marco de ciudades de escala intermedia comunes en el contexto latinoamericano. 
Limites y fragmentos en los territorios de la expansión:

la suburbanización entre las ciudades de Santa Fe y Santo Tomé

Las limitaciones espaciales manifiestan la búsqueda de cierta homogeneidad y seguridad, aparentes y artificiales, donde los lazos sociales son condicionados y forzados en detrimento de los múltiples intercambios que nutren la vida en las ciudades. En consecuencia, estos límites traducidos en dispositivos físicos y construidos para separar y aislar retroalimentan el sentimiento de inseguridad emergente en un entorno de violencia social tanto real como imaginada (KESSLER, 2009).

La observación de los límites temporales, enfocada en el valor tiempo como variable de análisis, pone en evidencia que un tratamiento ineficiente y parcial de la movilidad urbana, en tanto experiencia socio-cultural, puede convertirse en mecanismo de exclusión y desigualdad. En este sentido, la planificación debiera prestar atención a las múltiples prácticas que nutren la movilidad cotidiana urbana desalentando las movilidades segregadas y limitadas a determinados recorridos y medios de locomoción.

Por último, el análisis de límites sociales enfocado en el acceso diferencial al suelo urbano demuestra la fuerte incidencia que tienen las políticas urbanas en los procesos de fragmentación espacial y pone en evidencia la urgencia de instrumentos de control, y al mismo tiempo de cooperación conjunta, con los agentes involucrados en la mercantilización del suelo. A partir de estas lecturas, la noción de límite como instrumento metodológico para el análisis de la fragmentación espacial aporta al estudio de la seguridad, movilidad y economía urbana, en tanto problemáticas complejas de los territorios contemporáneos. 


\section{Bibliografía}

ASOCIACIÓN ARGENTINA DE CARRETERAS (1966). "Provincia de Santa Fe”. En Revista Carreteras. Asociación Argentina de Carreteras, Buenos Aires.

BAGNERA, Paola (2010) Arroyo Leyes. Planificación Urbana y Desarrollo Turístico. Ediciones UNL, Santa Fe.

BORSDORFT, Axel (2003). "Hacia la ciudad fragmentada. Tempranas estructuras segregadas en la ciudad latinoamericana”. En Scripta Nova VII 146 (122). Revista electrónica de geografía y ciencias sociales. Universidad de Barcelona, Barcelona. Recuperado en http://www.ub.es/ geocrit/sn/sn-146(122).htm.

BURGESS, Road (Julio, 2005). Technological determinism and urban fragmentation: A critical analysis. Trabajo presentado en 9th International Conference of the ALFA-IBIS Network on Urban Peripheries. 137-137. Pontificia Universidad Católica de Chile, Santiago de Chile. Recuperado en http://ccs.ukzn.ac.za/files/burgess\%20against\%20technological\%20 determinism.pdf.

CABRALES BARAJA, Luis Felipe y CANOSA ZAMORRA, Elia (2001). "Segregación residencial y fragmentación urbana: los fraccionamientos cerrados en Guadalajara”. En Espiral. Estudios sobre Estado y Sociedad VII (20), 223-253. Universidad de Guadalajara, México. Recuperado en http://www.revistascientificas.udg.mx/index.php/EEES/article/view/1191/1079.

CAPRON, Guénola y ESQUIVEL HERNÁNDEZ, María Teresa (2016). "El enclave urbano, lógica socioespacial de la periferia urbanizada y sus efectos sobre la segregación residencial y la fragmentación urbana”. En Cuadernos de Geografía. Revista colombiana de geografía. Universidad Nacional de Colombia, Bogotá. Recuperado en https://doi.org/10.15446/rcdg.v25n2.54720.

CARMAN, María, VIEIRA, Neira y SEGURA, Ramiro (2013) (coords). Segregación y diferencia en la ciudad. CLACSO, Ecuador.

COLLADO, Adriana (2012) El Corredor de la Costa. Conformación del paisaje y reconocimiento de sus recursos naturales. Ediciones UNL, Santa Fe.

CRUZ, Manuel (2013). Escritos sobre la ciudad y alrededores. Prometeo Libros, Buenos Aires. CUENYA, Beatriz (2016). "La política urbana frente a la mercantilización y elitización de la ciudad: algunas reflexiones y referencias a la situación argentina”. En Cuaderno Urbano, 21. pp. 167-194.

DIARIO EL LITORAL (1975). "La tatenguita: conjunto residencial-deportivo de novedosa y moderna concepcion urbanística y práctica”. Santa Fe, 3 de noviembre de 1975.

GÓMEZ, Néstor (2011). "Segregación residencial en el Gran Santa Fe a comienzos del siglo 
Limites y fragmentos en los territorios de la expansión:

la suburbanización entre las ciudades de Santa Fe y Santo Tomé

XXI”. Revista Bitácora Urbano Territorial, 19 (2). 63-73. Universidad Nacional de Colombia Bogotá, Colombia.

HIDALGO, Rodrigo (2004). "De los pequeños condominios a la ciudad vallada: las urbanizaciones cerradas y la nueva geografía social en Santiago de Chile (1990-2000)”. En EURE. Revista de Estudios Urbano Regionales 30 (91). 29-52.

JANOSCHKA, Michael (2002). "El nuevo modelo de la ciudad latinoamericana: fragmentación y privatización”. En EURE, 28 (85), 11-29. Recuperado en http://www.scielo.cl/scielo. php?script=sci_arttext\&pid=S0250-71612002008500002.

(2004). "El modelo de ciudad latinoamericana. Privatización y fragmentación del espacio urbano de Buenos Aires: el caso Nordelta”. En Welch Guerra, M. (Ed.). Buenos Aires a la deriva. Transformaciones urbanas recientes. (pp. 80-116). Biblios, Buenos Aires.

KESSLER, Gabriel (2009) El sentimiento de inseguridad. Sociología del temor al delito. Siglo Veintiuno Editores, Buenos Aires.

MARCUSE, Peter (1995). "No caos, sino muros: El postmodernismo y la ciudad compartimentada”. En Ramos, A. M. (ed.). Lo urbano en 20 autores contemporáneos. (pp. 83-90). Ediciones UPC, Barcelona.

PALLUD, Pamela (2010). "Las Urbanizaciones Privadas Cerradas en Santo Tomé, Santa Fe”. En Pampa. Revista Interuniversitaria de Estudios Territoriales (6). 37-58. Ediciones UNL, Santa Fe. PIRES DO RÍO CALDEIRA, Teresa (2007) Ciudad de muros. Gidesa, Barcelona.

RAUSCH, Gisela (2005). "Ilusiones territoriales. Las imágenes en la construcción del territorio costero santafesino”. En boletín de estudios geográficos (100), 31-48. Recuperado en http:// bdigital.uncu.edu.ar/objetos_digitales/5521/002rausch-beg-100.pdf.

RUEDA, Salvador (1997). "La ciudad compacta y diversa frente a la conurbación difusa. La construcción de la ciudad sostenible”. En Boletín Ciudades para un Futuro más Sostenible. Recuperado en http://habitat.aq.upm.es/cs/p2/a009.html.

SALINAS VARELA, Edison (2009). "Fragmentación urbana y su relevancia en la planificación urbana y territorial actual”. En Ignire. Centro de estudio de Política Pública. 49-58. Recuperado en http://www.ignire.cl/index.php/articulos2/40-16.

SASSEN, Saskia (1991) La Ciudad Global: Nueva York, Londres y Tokio. Eudeba, Buenos Aires. SVAMPA, Maristella (2004) La brecha urbana. Countries y barrios privados. Capital intelectual, Buenos Aires.

VAPÑARSKY, César y GOROJOVSKY, Néstor (1990). El crecimiento urbano en la Argentina. Grupo Editor Latinoamericano, Buenos Aires. 